\title{
Verzeichnis der hauptsächlichsten, für das Bergschädenrecht erheblichen Berggesetze, Novellen und Ausführungsgesetze.
}

Anhalt. B.G. vom 3o. April r875 §§ r37-15I XVI 28I. Neue Redaktion desselben vom 20. März 1896 Ges. Sammlung No. 960. A.G. zum B.G.B. vom 18. April 1899. Art. 53. Becher I 24; Art. zum R.Zv.G. vom 20. April 1899 Art. 12-18 Becher I 55.

Baden. B.G. vom 22. Juli $1890 \mathrm{XXXI}_{4} 83 \mathrm{ff}$. Nachtragsgesetz vom 22. Juni 1900 Gesetzs. S. 945.

Bayern. B.G. vom 20. März 1869 X $17 \mathrm{r}$ ff. ist am 3o. Juni 1900 in neuer Fassung veröffentlicht. XLIII 269 ff. Art. I84-r 88 .

Birkenfeld. B.G. vom 18 . März 1891 XXXIII 48 ff. Nachtragsgesetz dazu vom 27. Dezember 1899 (Gesetzesblatt fur Birkenfeld S. 403).

Braunschweig. B.G. vom r5. April $1867 \$$ r52-156 VIII $273 \mathrm{ff}$. Ges. vom 16. April 1892 betr. den Grundeigentümerbergbau XXXIII 279. Außerdem ist in Braunschweig das Gesetz vom 12. Juni 1899 betr. Abänderung des B.G. ergangen. XLI $3 \mathrm{r} 9 \mathrm{ff}$.

Coburg-Gotha, B.G. vom 23. Oktober 1899 §§ $166-170$ XLI $158 \mathrm{ff}$.

Elsa B-Lothringen. B.G. fur E.-L. vom 16. Dezember 1873 \$§ 127-131 $\mathrm{XV} 2 \mathrm{ff}$.

Hessen. B.G. vom 28. Januar 1876 Art. $14 \mathrm{I}-145$ XVII $158 \mathrm{ff}$. Hessisches A.G. zum B.G.B. vom 17. Juli 1899 Art. 283. Auf Grund des Art. 290 des A.G. ist das B.G. vom 28. Januar 1876 am 30 . November 1899 in neuer Fassung veröffentlicht. XLI $4 \mathrm{I} 5 \mathrm{ff}$.

Lippe-Detmold. Bergordnung vom 30. September 1857 III. der Gesetzsammlung für Fürstentum Lippe 7 r $5 \mathrm{ff}$.

Lübeck. B.G. vom 28. Oktober $1895 \$ \S$ 157-I6r XXXVII 42 Iff. Nachtragsgesetz vom 22. Dezember I 899 (Sammlung der Verordnungen Libecks Nr. 92).

Mecklenburg-Schwerin. Verordnung vom r6. Mai 1879 \& 4. Gesetz vom 19. Juni 1896 betr. die Gewerkschaften. XXXVII $439 \mathrm{ff}$.

Meiningen. B.G. vom 17. April I868 IX 315 ff. Nachtragsgesetz dazu vom II. August 1869. (Sammlung der Landesherrlichen Verordnungen S. 392.)

Preußen. Allg.B.G. vom 24. Juni 1865 VI 235. Einf.-Verordnung für Nassau vom 22. Februar 1867 VIII I. Einf.-Verordnung für Ober- 
hessen etc. vom 22. Felsruar 1867 VIII 29. Einf.-Verordnung für Hannover rom 8. Mai 1867 VIII 145. Einf.-Verordnung für Kurhessen etc. vom I. Juni 1867 VIII 197. Einf.-Gesetz für Lauenburg vom 6. Mai 1868 IX 289. Einf.-Gesetz für Schleswig-Holstein rom 12. März $1869 X$ 137. Einf. in das Jadegebiet (Ges, vom 23. März I873) XIV 324. Gesetz rom 22. Februar 1869, Stein- und Braunkohlenbergbau im Bezirke des kurf.-sächs. Mandats X 115. Gesetz rom 8. April 1894, Abänderung des § 21 I B.G. (Eisenerze in Schlesien). Gesetz vom 14. Juli 1895 , Salzbergbau in der Provinz Hannover XXXVI. 420 .

Reuß ä. L. Verordnung rom I. April 1897 betr. den Bergbau. XXXVIII $280 \mathrm{ff}$.

Reuß j. L. B.G. vom 9. Oktober 1870 §§ 102-IO5 XII 278 ff. Gewerkschaftsgesetz rom 29. März I895 XXXVIII $176 \mathrm{ffi}$, A.G. zum B.G.B. vom Io. August I 899 Becher II Iff. $§ 66$ A.G. zum R.Zv.G. vom Io. August 1899 , Becher II $87 \mathrm{ff}$. § I5-25.

$\mathrm{S}$ chaumburg-Lippe. Gesetz rom 12. Dezember 1872 betr. den Betrieb der Kohlenwerke XIV $298 \mathrm{ff}$. hat die preußische Bergschädenklage auf den Betrieb des Kohlenwerkes, welches für gemeinsame Rechnung des Schaumburgischen Fürstenhauses und des Königl. Preuß. Bergfiskus gefthrt wird, ausgedehnt.

Schwarzburg-Rudolstadt. A.B.G. vom 20. März $1894 \$ \S$ I 58 ff. XXXVII 391 ff. A.G. zum B.G.B. rom I1. Juli 1899 Art. 125. Becher II XXIII 5 ff. Art. 25 A.G. zum R.Zv.G. vom 28. Februar 19oo. Becher a. a. O. S. $106 \mathrm{ff}$. Art. $12-17$.

Schwarzburg-Sondershausen. B.G. vom 6. März $1894 \$ \S 158-163$ XXXVII 26r ff. A.G. zum B.G.B. vom 19. Juli 1899. Becher II XXIV I ff. Art. 44 A.G. zum R.Zv.G. vom 19. Juli 1899. Becher a. a. O. S. 44 ff. $\S \S 9-1$ 3. A.G. vom 29. Juli 1 899 . Becher a. a. O. S. 89 ff. § 33 .

Sacbsen-Altenburg. A.B.G. vom 18. April I872 $\$ \S 14 I-145$ B.G.B. XIII $156 \mathrm{ff}$. Gesetz über den Kohlenbergbau von demselben Tage. XIII 169 ff. Becher II XVIII I ff. \$ 52 A.G. zur R.Zv.O. vom 4. Mai 1898. Becher a. a. O. S. 4 I ff. $\$ \S 6-17$.

Sachsen-IVeimar. Gesetz über den Bergbau rom 22. Juni 1857 . Weimarsches Regierungsblatt von 1857 S. $149 \mathrm{ff}$.

Königreich Sachsen. A.B.G. vom I6. Juni 1868 IX $488 \mathrm{ff}$. Dannenberg S. 3 ff. A.G. zum R.Zv,G. vom 5. Dezember 1898 . Becher a. a. O. II XVII $102 \mathrm{ff}$. 29.

Waldeck-Pyrmont. Ges. vom 1. Januar 1864 betr. Einführung des Preuß. A.B.G. X 143 A.G. zum R.Zr.G. rom Ir. Dezember 1899. Becher a. a. O. S. 39 ff. Art. 13-25.

Württemberg. B.G. vom 7. Oktober 1874 Art. $136 \mathrm{ff}$. XV 431 ff. A.G. zum B.G.B. und den Nebengesetzen vom 28. Juli 1899 Art. 207 XLI $404 \mathrm{ff}$. 
